\title{
Associations Between Activities Outside Work and Presenteeism Among Korean Wage Workers: An Analysis Using a Nationwide Survey
}

\author{
Sung Won Jung, June-Hee Lee, Kyung-Jae Lee \\ Department of Occupational and Environmental Medicine, Soonchunhyang University Hospital, Seoul, Korea
}

Objectives: Presenteeism is currently recognized as a significant global health issue that can potentially cause productivity losses. Hence, many studies have analyzed the relationships between workplace factors and presenteeism. However, few studies have considered non-occupational factors. This study examined the associations between presenteeism and activities outside work, including volunteering, self-development, leisure/sports, and gardening and house repair activities, in Korean wage workers.

Methods: This study analyzed the fourth Korean Working Conditions Survey, in which a total of 19294 wage workers participated. To identify relationships between presenteeism and activities outside work, multivariate logistic regression analysis was used after adjusting for general and occupational characteristics.

Results: Self-development and leisure/sports activities significantly increased the odds ratio (OR) of presenteeism (OR, 1.166; 95\% confidence interval [Cl], 1.061 to 1.282 and $\mathrm{OR}, 1.276 ; 95 \% \mathrm{Cl}, 1.181$ to 1.379 , respectively).

Conclusions: Certain activities outside work, such as self-development or leisure/sports, were related to presenteeism among Korean wage workers. Although many previous studies have emphasized the positive effects of those activities on health, this study documented negative effects of these activities outside work on health.

Key words: Presenteeism, Activities outside work, Working conditions survey, Wage worker, Korea

\section{INTRODUCTION}

Modern-day wage workers spend half their lives at work. Therefore, workplace health has become an important issue, for both employees and employers. Health problems in work-

Received: May 31, 2019 Accepted: July 12, 2019

Corresponding author: Kyung-Jae Lee, MD, PhD

Department of Occupational and Environmental Medicine,

Soonchunhyang University Hospital, 59 Daesagwan-ro, Yongsan-gu,

Seoul 04401, Korea

E-mail: leekj@schmc.ac.kr

This is an Open Access article distributed under the terms of the Creative Commons Attribution Non-Commercial License (http://creativecommons.org/licenses/by$\mathrm{nc} / 4.0 /$ ) which permits unrestricted non-commercial use, distribution, and reproduction in any medium, provided the original work is properly cited. ers are associated with productivity losses, which are directly linked to the employers' interests [1], and the indirect economic burden caused by health-related productivity losses has been reported to be greater than the economic burden caused by other factors [2]. Presenteeism is quickly gathering attention as an issue that is relevant to productivity losses, and it is currently the focus of various ongoing studies. Presenteeism is the opposite concept of absenteeism. Previous studies have presented various definitions of presenteeism, including limitations of an individual's work efficiency despite coming into work [3] as a more general definition, as well as more specific definitions that focus on limitations of work efficiency due to coming into work with health problems [4,5]. Although some definitional confusion persists, the most recent scholarly 
conception of presenteeism involves showing up at work despite being ill [6].

Studies have suggested that the productivity and work attendance of workers with health problems decrease over time [7]. Other studies have investigated the impact of presenteeism on health. One such study found that workers who worked for 3 consecutive years had a higher rate of cardiovascular illnesses than workers who took time off [8]. When examining workers with experiences of presenteeism, it was noted that they assessed their own future health as deficient $[9,10]$. According to a Swedish study, at least one-third of workers experience presenteeism [5]. Likewise, in Korea, the percentage of workers who had experienced presenteeism was reported to be $21 \%$ according to the third Korean Working Conditions Survey (KWCS), underscoring workplace health as an important issue [11].

Presenteeism is significantly affected by both organizational and personal factors [12]. Some research has been conducted to confirm the relationships between presenteeism and organizational factors, such as psychological and occupational factors in the workplace [13-15], and individual health risk factors such as smoking, alcohol consumption, and physical exercise $[16,17]$. Activities outside work, such as physical exercise, provide an excellent opportunity to restore an individual's fitness, and to help control individual health risk factors [18]. Activities such as leisure sports, physical exercise, and gardening may diminish the symptoms of depression and anxiety, while improving sleep quality and other psychological factors $[18,19]$. The health events caused by chronic or acute illness affect work production and workers' attitudes [6]. In particular, chronic diseases have a strong relationship with activities outside work, and consequently, have an indirect effect on presenteeism [20].

Workers' health problems, as a personal factor, are directly linked to presenteeism, and individual health problems are inevitably associated with various activities outside work. However, few studies have yet examined the relationships between activities outside work and presenteeism. Furthermore, the existing research has mainly focused on the relationship between physical exercise and presenteeism, without considering a more diverse range of activities outside work $[21,22]$. Therefore, this study explored the relationships between presenteeism and various kinds of activities undertaken by Korean wage workers outside work utilizing data from the KWCS.

\section{METHODS}

\section{Study Subjects}

This research utilized resources from the fourth KWCS, conducted by the Korea Occupational Health and Safety Agency in 2014. The KWCS was developed based on the European Working Conditions Survey.

The subjects of the survey were employees over the age of 15 , and a total of 50007 people were interviewed and surveyed for the fourth KWCS. Participants who gave incomplete answers such as "I don't know/No answer" or refused to participate were removed from the survey results. This study was aimed at wage workers; thus, the responses of self-employed entrepreneurs, business owners, unpaid workers in family businesses, and other ineligible subjects were removed. Additionally, the responses of a few people who identified themselves as soldiers, agricultural workers, forestry workers, and fishery workers, as well as those of underage participants (15-20 years), were removed. Finally, participants with preexisting injuries, experience of harmful accidents, and previous cardiovascular issues potentially influencing an individual's experience of presenteeism were removed. In total, this study analyzed data from 19294 wage earners above the age of 20 .

\section{Measurements}

\section{General characteristics}

Participants' general characteristics included sex, age $(<30$ years, 30-39 years, 40-49 years, 50-59 years, $\geq 60$ years), and educational level (middle school or lower, high school diploma, university graduate, and other).

\section{Occupational characteristics}

Occupational characteristics included employment status (regular work, temporary work, or day labor), occupation type (management/professional, office work, service/sales, technical, or simple labor), weekly working hours ( $\leq 40,41-52,>52$, categorized based on the Labor Standard Act of Korea), shift work, number of employees at the workplace $(<5,5-49,50-299$, or $\geq 300$, categorized based on the Korean Industrial Safety and Health Act), and monthly income $(<1300000$ Korean won [KRW], 1300 000-1 990000 KRW, 2000 000-2 990000 KRW or $\geq 3000000 \mathrm{KRW}$ ). Monthly income was analyzed using the post-tax amount and categorized by quartile [23]. 


\section{Activities outside work}

Typical examples of activities outside work include volunteering, self-development activities, gardening, house repair, cultural activities, and sports $[23,24]$. The items used to assess "activities outside work" acted as independent variables in this study. Participants were asked to respond to the question "In general, how often are you involved in any of the following activities outside work?" The options for activities outside work included volunteer work, self-development, sports, physical exercise, leisure activities, gardening, and house repair. If participants' responses included "more than an hour every day," "less than an hour every day or every two days," "once or twice a week," or "once or twice a month," they were considered to have participated in that activity [23].

\section{Presenteeism}

Presenteeism can be defined as workers coming to work even when they should be resting at home to recover from illness or injury $[5,6]$. Thus, the study identified participants with an experience of presenteeism as the dependent variable based on an answer of "yes" to the question "Over the past 12 months, did you work despite being sick on at least one occasion?"

\section{Data Analysis}

The chi-square test was conducted to evaluate the statistical significance of differences in the general and occupational characteristics of wage workers who had experienced presenteeism, and to assess the distribution of presenteeism in relation to activities outside work. Next, correlations between activities outside work (the independent variables) and presenteeism (the dependent variable) were analyzed. General and occupational characteristics were adjusted and run through multiple logistic regression. The level of significance was set at $p$-value $<0.05$ and all statistical analyses were conducted using SPSS version 25.0 (IBM Corp., Armonk, NY, USA).

\section{Ethics Statement}

The KWCS comprises national open-source data with safeguards to protect the participants' anonymity and privacy rights, the requirement for institutional review board approval was not applicable to this study.

Table 1. Number of workers with presenteeism by general and occupational characteristics

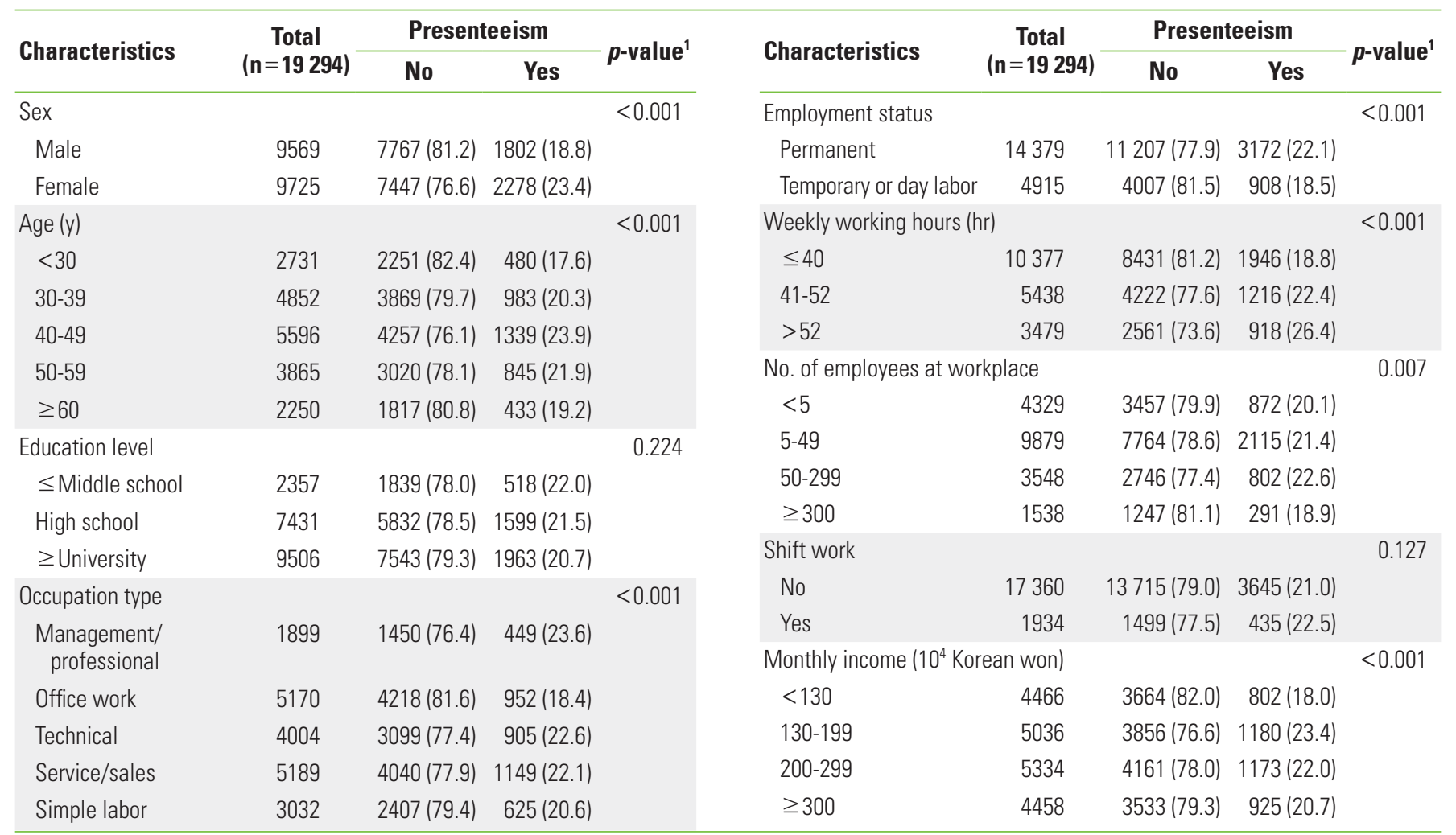

Values are presented as number or number (\%).

${ }^{1}$ Based on the chi-square test. 


\section{RESULTS}

\section{Distribution of Presenteeism With Regard to General and Occupational Characteristics}

The chi-square test was used to analyze the distribution of presenteeism with regard to general and occupational characteristics (Table 1). More female (23.4\%) had experienced presenteeism than male (18.8\%). More wage workers in the 40-49 age group (23.9\%) had experienced presenteeism than those in other age groups. Workers in professional and managerial jobs (23.6\%) were more likely to have experienced presenteeism than participants of other occupational types, and permanent workers (22.1\%) experienced more presenteeism than temporary/non-permanent workers (18.5\%). Those who worked over 52 hours a week were more likely to have experienced presenteeism (26.4\%), suggesting that presenteeism increased with a higher number of working hours. In workplaces with 50-299 workers, $22.6 \%$ of workers had experienced presenteeism, which was higher than the corresponding proportions in the workplaces with less than 5, 5-49, or more than 300 staff. Educational level and shift work showed no significant relationships with presenteeism.

\section{Distribution of Presenteeism With Regard to Activities Outside Work}

The chi-square test was conducted to evaluate the distribution of presenteeism with regard to various activities (Table 2). More workers who engaged in self-development activities had experienced presenteeism (23.0\%) than those who did not. Presenteeism in subjects who engaged in leisure and sports activities was higher than in those who did not (22.6\%). The results did not show any significant relationships between presenteeism and engaging in volunteering and gardening and house repair activities.

\section{Relationship Between Activities Outside Work and Presenteeism}

Simple and multivariate logistic regression analysis was performed to determine the relationship between presenteeism and activities outside work (Table 3). The previously mentioned general and occupational characteristics were adjusted

Table 2. Distribution of presenteeism by activities outside work

\begin{tabular}{lrrrr}
\hline & \multirow{2}{*}{$\begin{array}{c}\text { Total } \\
\text { Variables }\end{array}$} & \multicolumn{2}{c}{ Presenteeism } & \\
& (n=19 294) & \multicolumn{1}{c}{ No } & Yes & \\
\hline Volunteering & & & & 0.800 \\
$\quad$ None & 18443 & $14540(78.8)$ & $3903(21.2)$ & \\
$\quad$ Participated & 851 & $674(79.2)$ & $177(20.8)$ & \\
Self-development & & & & 0.001 \\
$\quad$ None & 15457 & $12261(79.3)$ & $3196(20.7)$ & \\
$\quad$ Participated & 3837 & $2953(77.0)$ & $884(23.0)$ & \\
Leisure and sports & & & & $<0.001$ \\
$\quad$ None & 8353 & $6741(80.7)$ & $1612(19.3)$ & \\
$\quad$ Participated & 10941 & $8473(77.4)$ & $2468(22.6)$ & \\
Gardening and house repair & & & & 0.241 \\
$\quad$ None & 16301 & $12878(79.0)$ & $3423(21.0)$ & \\
$\quad$ Participated & 2993 & $2336(78.0)$ & $657(22.0)$ & \\
\hline
\end{tabular}

Values are presented as number or number (\%).

${ }^{1}$ Based on the chi-square test.

Table 3. Relationship between activities outside work and presenteeism

\begin{tabular}{|c|c|c|c|}
\hline Variables & Model I ${ }^{1}$ & Model II² & Model III \\
\hline \multicolumn{4}{|l|}{ Volunteering } \\
\hline None & 1.000 (reference) & 1.000 (reference) & 1.000 (reference) \\
\hline Participated & $1.087(0.915,1.291$ & $1.037(0.873,1.234)$ & $0.899(0.755,1.071)$ \\
\hline \multicolumn{4}{|c|}{ Self-development } \\
\hline None & 1.000 (reference) & 1.000 (reference) & 1.000 (reference) \\
\hline Participated & $1.083(0.990,1.185)$ & $1.239(1.132,1.357)$ & $1.166(1.061,1.282)$ \\
\hline \multicolumn{4}{|l|}{ Leisure/sports } \\
\hline None & 1.000 (reference) & 1.000 (reference) & 1.000 (reference) \\
\hline Participated & $1.198(1.112,1.291)$ & $1.306(1.212,1.406)$ & $1.276(1.181,1.379)$ \\
\hline \multicolumn{4}{|c|}{ Gardening and house repair } \\
\hline None & 1.000 (reference) & 1.000 (reference) & 1.000 (reference) \\
\hline Participated & $0.998(0.906,1.100)$ & $1.063(0.966,1.171)$ & $0.979(0.887,1.081)$ \\
\hline
\end{tabular}

Values are presented as odds ratio (95\% confidence interval).

${ }^{1}$ Model I: crude odds ratios.

${ }^{2}$ Model II: simple logistic regression analysis, adjusting for general and occupational characteristics simultaneously.

${ }^{3}$ Model III: multivariate logistic regression analysis, adjusting for general and occupational characteristics simultaneously. 
and analyzed in model II and III. The results showed that the risk of presenteeism was higher in workers who participated in self-development activities than in those who did not (odds ratio [OR], 1.166; 95\% confidence interval [Cl], 1.061 to 1.282). An elevated risk of presenteeism was also apparent in workers who participated in leisure and sports activities compared to those who did not (OR, 1.276; $95 \% \mathrm{Cl}, 1.181$ to 1.379 ).

\section{DISCUSSION}

This study examined the relationships between activities outside of work among Korean wage workers (volunteering, self-development, leisure and sports activities, and gardening and house repairs) and the experience of presenteeism. The study found that the risk of presenteeism was notably elevated in individuals who participated in self-development activities or leisure and sport activities.

Our study showed that participating in self-development activities increased the risk of presenteeism. A previous study that used the fourth KWCS found that self-development activities increased the risk of sleep disorders [23]. The aforementioned study concluded that the self-development activities undertaken by Korean workers were not for self-satisfaction, but rather were considered as a means to gain promotion or secure employment, which can be a cause of stress. Other studies have identified individuals suffering from insomnia and sleep disorders as vulnerable to presenteeism [25], and these results correlate with our research findings. Another possible mechanism underlying our findings is that because Korean workers' self-development activities commonly occur in offwork hours for personal gain, they limit the availability of time for rest [26].

Many studies have examined the effects of sports, leisure, and physical activity on health, and most have reported positive results. It is well established that participating in leisure activities, physical activity, and sports can lower the risk of obesity [27] and cardiovascular diseases [28]. Past studies have shown that obesity and cardiovascular diseases can raise the risk of presenteeism [29]. We expected leisure and sports activities to lower the risk of presenteeism, in line with previous research, but the results of this study indicated the opposite tendency.

In previous research, leisure and physical activities were shown to have harmful effects on mortality and risk of cardiovascular diseases when coupled with high levels of occupa- tional physical activity [30,31]. Korean workers are among the hardest-working in the Organization for Economic Cooperation and Development, and are known for their high rates of occupational physical load and work intensity. In a previous study on Korean wage workers, over $70 \%$ of workers were exposed to occupational physical activities such as painful or tiring positions, repetitive hand or arm movements, moving or lifting heavy loads, and standing for long hours, and these workers may be more susceptible to presenteeism [11]. It is possible that these factors may have influenced our research results. Further, involuntary leisure and physical activities embedded into Korea's work culture, such as company sports days, weekend hikes, and other after-work activities, may also have contributed to the results.

The main strength of this study is that it is the first of its kind to investigate the relationships of activities outside work, such as volunteering, self-development, leisure/sports, and gardening, with presenteeism. Through this study, we have provided an opportunity to consider the health effects of some activities outside work. Furthermore, this study suggests that the off-work hours of Korean wage workers may be seen an extension of work through self-development activities, indicating that this may be an example of how the competitive work culture in Korea has adverse health effects. Such findings highlight the need for additional research to closely examine the work environment in Korea.

The study has certain limitations. First, as a cross-sectional study, it did not reveal causal relationships between certain activities outside work and presenteeism. Nonetheless, our study presents some novel relationships, making it meaningful in the sense that new possibilities are presented. Second, because of the nature of the KWCS data, categorized self-written questionnaires were used, making it difficult to objectively evaluate activities outside work as an independent variable. In addition, we could not evaluate the dependent variable, presenteeism, in an objective manner through a tool such as the Stanford presenteeism scale. This factor may reduce the objectivity of this study. However, many existing studies using selfreport questionnaires or the KWCS data have reported sufficient validity, so the findings of this study are still sufficiently valuable. Despite the limitations outlined above, the results of this study constitute a valuable resource for further research to find new solutions to issues facing Korean wage workers.

This study showed that certain kinds of activities outside work, such as self-development and leisure/sports activities, 
increased the risk of presenteeism in Korean wage workers. The results of this study indirectly suggest that, for Korean wage workers, after-work hours are merely seen as an extension of work, a unique characteristic of Korea's work culture that may be a cause of presenteeism. In conclusion, further research examining the specific components of Korea's unique work culture is necessary.

\section{CONFLICT OF INTEREST}

The authors have no conflicts of interest associated with the material presented in this paper.

\section{ACKNOWLEDGEMENTS}

We express our appreciation for the Statistics Team of the Occupational Safety and Health Research Institute (OSHRI) for providing the raw data from the Korean Working Conditions Survey (KWCS).

This work was supported by the Soonchunhyang University Research Fund.

\section{AUTHOR CONTRIBUTIONS}

Conceptualization: SWJ, JHL. Data curation: SWJ. Formal analysis: SWJ, JHL. Funding acquisition: KJL. Methodology: SWJ, JHL, KJL. Project administration: JHL, KJL. Visualization: JHL, KJL. Writing - original draft: SWJ. Writing - review \& editing: SWJ, JHL, KJL.

\section{ORCID}

Sung Won Jung https://orcid.org/0000-0001-5668-2459 June-Hee Lee https://orcid.org/0000-0002-7682-6023 Kyung-Jae Lee https://orcid.org/0000-0001-5759-2367

\section{REFERENCES}

1. Loeppke R, Taitel M, Haufle V, Parry T, Kessler RC, Jinnett K. Health and productivity as a business strategy: a multiemployer study. J Occup Environ Med 2009;51(4):411-428.

2. Goetzel RZ, Long SR, Ozminkowski RJ, Hawkins K, Wang S, Lynch W. Health, absence, disability, and presenteeism cost estimates of certain physical and mental health conditions affecting U.S. employers. J Occup Environ Med 2004;46(4):398-
412.

3. Schultz AB, Edington DW. Employee health and presenteeism: a systematic review. J Occup Rehabil 2007;17(3):547-579.

4. Kivimäki M, Head J, Ferrie JE, Hemingway H, Shipley MJ, Vahtera J, et al. Working while ill as a risk factor for serious coronary events: the Whitehall II study. Am J Public Health 2005; 95(1):98-102.

5. Aronsson G, Gustafsson K, Dallner M. Sick but yet at work. An empirical study of sickness presenteeism. J Epidemiol Community Health 2000;54(7):502-509.

6. Johns G. Presenteeism in the workplace: a review and research agenda. J Organ Behav 2010;31(4):519-542.

7. Lamb CE, Ratner PH, Johnson CE, Ambegaonkar AJ, Joshi AV, Day $\mathrm{D}$, et al. Economic impact of workplace productivity losses due to allergic rhinitis compared with select medical conditions in the United States from an employer perspective. Curr Med Res Opin 2006;22(6):1203-1210.

8. Kivimäki M, Batty GD, Hamer M, Ferrie JE, Vahtera J, Virtanen M, et al. Using additional information on working hours to predict coronary heart disease: a cohort study. Ann Intern Med 2011; 154(7):457-463.

9. Bergström G, Bodin L, Hagberg J, Lindh T, Aronsson G, Josephson M. Does sickness presenteeism have an impact on future general health? Int Arch Occup Environ Health 2009;82(10): 1179-1190.

10. Gustafsson K, Marklund S. Consequences of sickness presence and sickness absence on health and work ability: a Swedish prospective cohort study. Int J Occup Med Environ Health 2011;24(2):153-165.

11. Jeon SH, Leem JH, Park SG, Heo YS, Lee BJ, Moon SH, et al. Association among working hours, occupational stress, and presenteeism among wage workers: results from the second Korean Working Conditions Survey. Ann Occup Environ Med 2014; 26(1):6.

12. Garrow V. Presenteeism: a review of current thinking; 2016 [cited 2019 May 31]. Available from: https://www.employment-studies.co.uk/system/files/resources/files/507_0.pdf.

13. Aronsson G, Gustafsson K. Sickness presenteeism: prevalence, attendance-pressure factors, and an outline of a model for research. J Occup Environ Med 2005;47(9):958-966.

14. de Graaf R, Tuithof M, van Dorsselaer S, ten Have M. Comparing the effects on work performance of mental and physical disorders. Soc Psychiatry Psychiatr Epidemiol 2012;47(11): 1873-1883.

15. Demerouti E, Le Blanc PM, Bakker AB, Schaufeli WB, Hox J. Pres- 
ent but sick: a three-wave study on job demands, presenteeism and burnout. Career Dev Int 2009;14(1):50-68.

16. Burton WN, Chen CY, Conti DJ, Schultz AB, Edington DW. The association between health risk change and presenteeism change. J Occup Environ Med 2006;48(3):252-263.

17. Serxner SA, Gold DB, Bultman KK. The impact of behavioral health risks on worker absenteeism. J Occup Environ Med 2001; 43(4):347-354.

18. Tucker $P$, Dahlgren A, Akerstedt T, Waterhouse J. The impact of free-time activities on sleep, recovery and well-being. Appl Ergon 2008;39(5):653-662.

19. Clatworthy J, Hinds J, Camic PM. Gardening as a mental health intervention: a review. Ment Health Rev J 2013;18(4):214-225.

20. Koopman C, Pelletier KR, Murray JF, Sharda CE, Berger ML, Turpin RS, et al. Stanford presenteeism scale: health status and employee productivity. J Occup Environ Med 2002;44(1):14-20.

21. Walker TJ, Tullar JM, Diamond PM, Kohl HW 3rd, Amick BC 3rd. Association of self-reported aerobic physical activity, musclestrengthening physical activity, and stretching behavior with presenteeism. J Occup Environ Med 2017;59(5):474-479.

22. Walker TJ, Tullar JM, Diamond PM, Kohl HW 3rd, Amick BC 3rd. The longitudinal relation between self-reported physical activity and presenteeism. Prev Med 2017;102:120-126.

23. Jung SW, Lee KJ, Lee HS, Kim GH, Lee JG, Lee JH, et al. Relationship of activities outside work to sleep and depression/anxiety disorders in Korean workers: the 4th Korean Working Condition Survey. Ann Occup Environ Med 2017;29:51.

24. Nasermoaddeli A, Sekine M, Kumari M, Chandola T, Marmot M, Kagamimori S. Association of sleep quality and free time lei- sure activities in Japanese and British civil servants. J Occup Health 2005;47(5):384-390.

25. Kucharczyk ER, Morgan K, Hall AP. The occupational impact of sleep quality and insomnia symptoms. Sleep Med Rev 2012; 16(6):547-559.

26. Hwang CY. A study on the relationship between organizational culture and performance management. Korean Policy Stud Rev 2003;12(2):283-310 (Korean).

27. Sarma S, Zaric GS, Campbell MK, Gilliland J. The effect of physical activity on adult obesity: evidence from the Canadian NPHS panel. Econ Hum Biol 2014;14:1-21.

28. Cheung YK, Moon YP, Kulick ER, Sacco RL, Elkind MS, Willey JZ. Leisure-time physical activity and cardiovascular mortality in an elderly population in northern Manhattan: a prospective cohort study. J Gen Intern Med 2017;32(2):168-174.

29. Gates DM, Succop P, Brehm BJ, Gillespie GL, Sommers BD. Obesity and presenteeism: the impact of body mass index on workplace productivity. J Occup Environ Med 2008;50(1): 39-45.

30. Hu GC, Chien KL, Hsieh SF, Chen CY, Tsai WH, Su TC. Occupational versus leisure-time physical activity in reducing cardiovascular risks and mortality among ethnic Chinese adults in Taiwan. Asia Pac J Public Health 2014;26(6):604-613.

31. Clays E, De Bacquer D, Janssens H, De Clercq B, Casini A, Braeckman $\mathrm{L}$, et al. The association between leisure time physical activity and coronary heart disease among men with different physical work demands: a prospective cohort study. Eur J Epidemiol 2013;28(3):241-247. 